\title{
Asymptotically Stable Observer-based Controller for Attitude Tracking with Systematic Convergence
}

\author{
Hashim A. Hashim
}

\begin{abstract}
This paper proposes a novel unit-quaternion observer-based controller for attitude tracking (attitude and angular velocity) with guaranteed transient and steady-state performance. The proposed approach is computationally cheap and can operate based on measurements provided, for instance by a typical low-cost inertial measurement unit (IMU) or magnetic, angular rate, and gravity (MARG) sensor without the knowledge of angular velocity. First, an observer evolved on $\mathbb{S}^{3} \times \mathbb{R}^{3}$ is developed guaranteeing asymptotic stability of the closed loop error signals starting from any initial condition. Afterwords, the observer is combined with the proposed controller such that the observer-based controller ensures asymptotic stability of the closed loop error signals starting from any initial condition. Simulation performed in discrete form at low sampling rate reveals the robustness and effectiveness of the proposed approach.
\end{abstract}

Index Terms-Observer-based controller, attitude, estimation, control, MARG, IMU, asymptotic stability.

\section{INTRODUCTION}

A TTITUDE tracking is a fundamental part of a variety of robotics applications including space telescopes, unmanned aerial vehicles, rotating radars and others. Development of cheap, small-sized, low-weight, and power-efficient inertial measurement units (IMUs) sparked a wave of active research in the area of attitude observation and tracking (observer + control) [1-11]. The main challenge of working with low-cost sensors, such as IMUs is their susceptibility to noise. Also, the true attitude dynamics rely on angular velocity of a rigid-body, commonly measured by a gyroscope. Nonetheless, replacement of a failed gyroscope often proves to be challenging and costly [12]. Hence, there is a need for effective attitude tracking solutions that do not require knowledge of angular velocity.

Velocity-free attitude control is possible with a full-state observer able to provide accurate estimates of both attitude and angular velocity. Thereafter, an attitude tracking control based solely on available attitude and angular velocity estimates is developed. It is worth mentioning that angular velocity is observable only given the knowledge of attitude. In turn, in order to obtain attitude information it is sufficient to acquire at least two vectorial measurements at the rigid-body using, for example, an IMU module [2,6,13-15]. An early solution presented a full state observer for rigid-body motion [1]. The problem of velocity-free attitude tracking has been addressed in the literature in a variety of ways including

This work was supported in part by Thompson Rivers University Internal research fund \# 102315.

${ }^{*}$ Corresponding author, H. A. Hashim is with the Department of Engineering and Applied Science, Thompson Rivers University, Kamloops, British Columbia, Canada, V2C-0C8, e-mail: hhashim@tru.ca. a full-state observer for rigid-body motion [1], an observerbased controller with local exponential stability [16], attitude tracking control without velocity measurements [17], and a hybrid attitude tracking controller with semi-global asymptotic stability which has a switching observer that restores angular velocity signal [4]. Additionally, an observer-based controller for the attitude tracking problem has been formulated to handle unknown bounded external disturbances $[18,19]$.

Despite a multitude of existing solutions, the common issue all of them share is the inability to guarantee the attitude error transient and steady-state performance. Lack of certainty and predictability in the performance of attitude observation and the control errors can easily destabilize the entire process. However, full control over the transient and steadystate performance can be gained by employing a prescribed performance function (PPF) [20]. PPF is able to guide the error to initiate within a large set and reduce systematically to settle within a small set. PPF approach has been successfully utilized for attitude-related problems, for example, an observer-based controller for attitude tracking problem subject to actuator saturation [21] and output feedback of attitude problem subject to external disturbances [22]. The work in [21] used angle-axis, whereas the work in [22] considered Rodriguez parameters for attitude parameterization. Both angle-axis and Rodriguez parameters approaches for attitude parameterization are subject to singularity. Moreover, the overall closed loop signals of [21,22] are shown to be semi-globally uniformly ultimately bounded, and therefore, the asymptotic stability cannot be guaranteed.

Considering the above literature overview, it becomes apparent that in order to achieve a stable attitude tracking process and alleviate the need for angular velocity information, observer-based control solutions with guaranteed measures of transient and steady-state performance of attitude error should be developed. Thus, the main contributions of this work are: 1) an attitude and angular velocity observer developed on $\mathbb{S}^{3} \times \mathbb{R}^{3}$ guaranteeing almost global asymptotic stability with predefined measures of transient and steady-state performance of attitude error is proposed. 2) The estimates of attitude and angular velocity obtained by the observer are combined with a novel attitude tracking control law that ensures almost global asymptotic stability with guaranteed measures of transient and steady-state performance of attitude error. 3) The proposed solutions produce accurate results even when supplied with uncertain measurements obtained from a low-cost IMU module at low sampling rate.

The rest of the article is organized as follows: Section II introduces the math notation, unit-quaternion preliminaries, attitude dynamics, available measurements, and attitude error. 
Section III presents the concept of PPF. Section IV introduces the observer-based controller for the attitude tracking problem. Section V demonstrates the robustness of the proposed approach through numerical results. Finally, Section VI summarizes the work.

\section{PRoblem Formulation}

\section{A. Preliminaries}

Let $\mathbb{R}$ and $\mathbb{R}^{n \times m}$ denote a set of real numbers and a real $n$-by- $m$ dimensional space, respectively. For $x \in \mathbb{R}^{n}$, $\|x\|=\sqrt{x^{\top} x}$ denotes the Euclidean norm. $\{\mathcal{I}\}$ and $\{\mathcal{B}\}$ correspond to fixed inertial-frame and body-frame, respectively. $R \in \mathbb{S O}(3)$ describes rigid-body's attitude (orientation) where $[2,6]$

$$
\mathbb{S O}(3)=\left\{R \in \mathbb{R}^{3 \times 3} \mid R R^{\top}=R^{\top} R=\mathbf{I}_{3}, \operatorname{det}(R)=+1\right\}
$$

with $\operatorname{det}(\cdot)$ being a determinant. Let $Q=\left[q_{0}, q^{\top}\right]^{\top} \in \mathbb{S}^{3}$ stand for a unit-quaternion vector where $q_{0} \in \mathbb{R}$ and $q \in \mathbb{R}^{3}$ such that $\mathbb{S}^{3}=\left\{Q \in \mathbb{R}^{4} \mid\|Q\|=\sqrt{q_{0}^{2}+q^{\top} q}=1\right\}$. $[\Omega]_{\times}$ represents a skew symmetric matrix with

$$
[\Omega]_{\times}=\left[\begin{array}{ccc}
0 & -\Omega_{3} & \Omega_{2} \\
\Omega_{3} & 0 & -\Omega_{1} \\
-\Omega_{2} & \Omega_{1} & 0
\end{array}\right], \quad \Omega=\left[\begin{array}{l}
\Omega_{1} \\
\Omega_{2} \\
\Omega_{3}
\end{array}\right]
$$

Note that $[\Omega]_{\times} y=\Omega \times y$ for all $\Omega, y \in \mathbb{R}^{3}$. Let $Q^{-1}=\left[q_{0},-q^{\top}\right]^{\top} \in \mathbb{S}^{3}$ be the inverse of $Q$. For $Q_{1}=$ $\left[\begin{array}{ll}q_{01} & q_{1}^{\top}\end{array}\right]^{\top} \in \mathbb{S}^{3}$ and $Q_{2}=\left[\begin{array}{ll}q_{02} & q_{2}^{\top}\end{array}\right]^{\top} \in \mathbb{S}^{3}$, the quaternion product is as follows:

$$
Q_{1} \odot Q_{2}=\left[\begin{array}{c}
q_{01} q_{02}-q_{1}^{\top} q_{2} \\
q_{01} q_{2}+q_{02} q_{1}+\left[q_{1}\right]_{\times} q_{2}
\end{array}\right] \in \mathbb{S}^{3}
$$

For $Q=\left[q_{0}, q^{\top}\right]^{\top} \in \mathbb{S}^{3}$, the related unit-quaternion mapping from $\mathbb{S}^{3}$ to $\mathbb{S O}(3)$ is as follows:

$$
\mathcal{R}_{Q}=\left(q_{0}^{2}-\|q\|^{2}\right) \mathbf{I}_{3}+2 q q^{\top}+2 q_{0}[q]_{\times} \in \mathbb{S O O}(3)
$$

In view of (1), the quaternion identity and its related mapping to $\mathbb{S O}(3)$ are defined by

$$
\mathbf{Q}_{\mathrm{I}}=[ \pm 1,0,0,0]^{\top} \quad \Leftrightarrow \quad \mathcal{R}_{\mathbf{Q}_{\mathrm{I}}}=\mathbf{I}_{3}
$$

For more details, visit [23,24]. For any $\Omega \in \mathbb{R}^{3}$ and $Q \in \mathbb{S}^{3}$, the following maps are considered:

$$
\begin{cases}\bar{\Omega} & =\left[0, \Omega^{\top}\right]^{\top} \in \mathbb{R}^{4} \\
\Gamma(\Omega) & =\left[\begin{array}{cc}
0 & -\Omega^{\top} \\
\Omega & -[\Omega]_{\times}
\end{array}\right] \in \mathbb{R}^{4 \times 4}\end{cases}
$$

\section{B. Measurements and Dynamics}

Let $Q \in \mathbb{S}^{3}$ and $\Omega \in \mathbb{R}^{3}$ denote the true unit-quaternion and angular velocity of a rigid-body in $3 \mathrm{D}$ space, respectively as depicted in Fig. 1. The true attitude dynamics on $\mathbb{S O}(3)$ are

$$
\dot{R}=R[\Omega]_{\times}, \quad J \dot{\Omega}=[J \Omega]_{\times} \Omega+\tau
$$

The equivalent unit-quaternion representation is as follows:

$$
\begin{cases}\dot{Q} & =\frac{1}{2} Q \odot \bar{\Omega}=\frac{1}{2} \Gamma(\Omega) Q \\ J \dot{\Omega} & =[J \Omega]_{\times} \Omega+\tau\end{cases}
$$

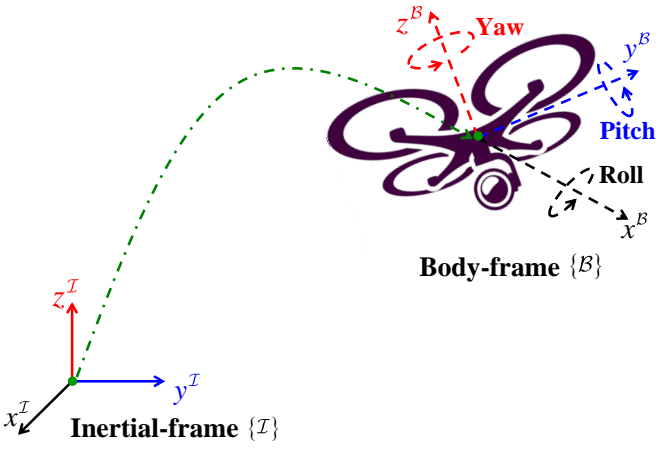

Fig. 1. Rigid-body's orientation (attitude) in body-frame $\{\mathcal{B}\}$ relative to inertial-frame $\{\mathcal{I}\}$

with $J$ being a positive-definite symmetric inertia matrix of a rigid-body, and $\tau \in \mathbb{R}^{3}$ being input torque. Note that $R, Q, \Omega, \tau, J \in\{\mathcal{B}\}$. A typical low-cost IMU module collects measurements using a 3-axis magnetometer and a 3-axis accelerometer at the body-frame $\{\mathcal{B}\}$ which can be expressed as follows:

$$
\overline{b_{i}}=Q^{-1} \odot \overline{r_{i}} \odot Q+\overline{n_{i}} \in \mathbb{R}^{4}, \quad \forall i=1,2, \ldots, n
$$

where $\overline{r_{i}}=\left[0, r_{i}^{\top}\right]^{\top} \in \mathbb{R}^{4}$ is the $i$ th known observation in the inertial frame $\{\mathcal{I}\}$ and $n_{i}$ is unknown noise. The vectors in (6) are normalized as follows:

$$
\mathfrak{r}_{i}=r_{i} /\left\|r_{i}\right\|, \quad \mathfrak{b}_{i}=b_{i} /\left\|b_{i}\right\|
$$

Let $M^{\mathcal{I}}=\left[\mathfrak{r}_{1}, \mathfrak{r}_{2}, \ldots, \mathfrak{r}_{n}\right]$ and $M^{\mathcal{B}}=\left[\mathfrak{b}_{1}, \mathfrak{b}_{2}, \ldots, \mathfrak{b}_{n}\right]$.

Remark 1. [2,6] The attitude can be reconstructed if $\left(\operatorname{rank}\left(M^{\mathcal{I}}\right)=\operatorname{rank}\left(M^{\mathcal{B}}\right)=3\right)$. For $n=2$, the third vector is defined by $\mathfrak{r}_{3}=\mathfrak{r}_{1} \times \mathfrak{r}_{2}$ and $\mathfrak{b}_{3}=\mathfrak{b}_{1} \times \mathfrak{b}_{2}$.

Let $Q_{y} \in \mathbb{S}^{3}$ denote a reconstructed quaternion of the true quaternion $Q \in \mathbb{S}^{3}$. $Q_{y}$ can be obtained by one of the methods of quaternion determination, for instance QUEST [13], optimal QUEST [25], or for others see [26]. The desired (reference) trajectory of $Q_{d} \in \mathbb{S}^{3}$ is defined by the desired angular velocity $\Omega_{d} \in \mathbb{R}^{3}$ as follows:

$$
\dot{Q}_{d}=\frac{1}{2} Q_{d} \odot \overline{\Omega_{d}}=\frac{1}{2} \Gamma\left(\Omega_{d}\right) Q_{d}, \quad Q_{d}(0) \in \mathbb{S}^{3}
$$

Assumption 1. Both $\Omega_{d}$ and $\dot{\Omega}_{d}$ are upper bounded by a scalar $C_{d}<\infty$ with $C_{d} \geq \max \left\{\sup _{t \geq 0}\left\|\Omega_{d}\right\|, \sup _{t \geq 0}\left\|\dot{\Omega}_{d}\right\|\right\}$.

Recall that the aim of this work is to design an observerbased controller characterized by guaranteed measures of transient and steady-state performance that does not require knowledge of angular velocity. Therefore, the first step consists in designing a full-state observer (attitude and angular velocity) evolved on $\mathbb{S}^{3} \times \mathbb{R}^{3}$ ensuring almost global asymptotic stability and following predefined measures of transient and steady-state performance of attitude observation error. Next, the full-state observer is combined with the attitude tracking control on $\mathbb{S}^{3}$ ensuring almost global asymptotic stability with guaranteed performance of attitude tracking error. The angular velocity is observable if the attitude is known. In view of Remark 1, the attitude, in turn, is observable if 
$\operatorname{rank}\left(M^{\mathcal{I}}\right)=\operatorname{rank}\left(M^{\mathcal{B}}\right)=3$. Let $\hat{Q}=\left[\hat{q}_{0}, \hat{q}^{\top}\right]^{\top} \in \mathbb{S}^{3}$, be the estimate of $Q=\left[q_{0}, q^{\top}\right]^{\top} \in \mathbb{S}^{3}$ and define the error between $Q$ and $\hat{Q}$ as

$$
\tilde{Q}_{o}=\left[\tilde{q}_{o 0}, \tilde{q}_{o}^{\top}\right]^{\top}=\hat{Q}^{-1} \odot Q \in \mathbb{S}^{3}
$$

Define $\hat{\Omega} \in \mathbb{R}^{3}$ as the estimate of $\Omega$ and let the error between $\Omega$ and $\hat{\Omega}$ be

$$
\overline{\tilde{\Omega}_{o}}=\bar{\Omega}-\tilde{Q}_{o}^{-1} \odot \overline{\hat{\Omega}} \odot \tilde{Q}_{o} \Leftrightarrow \tilde{\Omega}_{o}=\Omega-\mathcal{R}_{\tilde{Q}_{o}}^{\top} \hat{\Omega} \in \mathbb{R}^{3}
$$

where $\mathcal{R}_{\tilde{Q}_{o}}=\left(\tilde{q}_{o 0}^{2}-\left\|\tilde{q}_{o}\right\|^{2}\right) \mathbf{I}_{3}+2 \tilde{q}_{o} \tilde{q}_{o}^{\top}+2 \tilde{q}_{o 0}\left[\tilde{q}_{o}\right]_{\times}$, see the map in (1). Assume that $Q_{d}=\left[q_{d 0}, q_{d}^{\top}\right]^{\top} \in \mathbb{S}^{3}$ is the desired quaternion trajectory, and let the error between $Q$ and $Q_{d}$ be

$$
\tilde{Q}_{c}=\left[\tilde{q}_{c 0}, \tilde{q}_{c}^{\top}\right]^{\top}=Q_{d}^{-1} \odot Q \in \mathbb{S}^{3}
$$

Allow $\Omega_{d} \in \mathbb{R}^{3}$ to be the desired trajectory of angular velocity, and let the error between $\Omega$ and $\Omega_{d}$ be

$$
\overline{\tilde{\Omega}_{c}}=\bar{\Omega}-\tilde{Q}_{c}^{-1} \odot \overline{\Omega_{d}} \odot \tilde{Q}_{c} \Leftrightarrow \tilde{\Omega}_{c}=\Omega-\mathcal{R}_{\tilde{Q}_{c}}^{\top} \Omega_{d} \in \mathbb{R}^{3}
$$

where $\mathcal{R}_{\tilde{Q}_{c}}=\left(\tilde{q}_{c 0}^{2}-\left\|\tilde{q}_{c}\right\|^{2}\right) \mathbf{I}_{3}+2 \tilde{q}_{c} \tilde{q}_{c}^{\top}+2 \tilde{q}_{c 0}\left[\tilde{q}_{c}\right]_{\times}$. Recall (2), the objective of attitude observation is to drive $\tilde{Q}_{o} \rightarrow \mathbf{Q}_{\mathrm{I}}$ and $\tilde{\Omega}_{o} \rightarrow 0_{3 \times 1}$. Similarly, the objective of attitude control is to drive $\tilde{Q}_{c} \rightarrow \mathbf{Q}_{\mathrm{I}}$ and $\tilde{\Omega}_{c} \rightarrow 0_{3 \times 1}$, which, in turn, implies $\mathcal{R}_{\tilde{Q}_{o}} \rightarrow \mathbf{I}_{3}$ and $\mathcal{R}_{\tilde{Q}_{c}} \rightarrow \mathbf{I}_{3}$, visit (2). By the definition of unitquaternion and the identity property, $\tilde{q}_{o 0} \rightarrow \pm 1$ implies that $\tilde{q}_{o} \rightarrow 0_{3 \times 1}$ and vice versa. Likewise, $\tilde{q}_{c 0} \rightarrow \pm 1$ indicates that $\tilde{q}_{c} \rightarrow 0_{3 \times 1}$ and vice versa.

\section{Guaranteed Performance}

This section aims to guarantee that the tracking performance of

$$
e_{\star}=1-\tilde{q}_{\star 0}
$$

is initiated within a known large set and decreased smoothly to stay within a known small set where the subscript $\star$ is to be replaced by $o$ and $c$. Note that unit-quaternion is subject to non-uniqueness such that for $\tilde{Q}_{\star}=-\tilde{Q}_{\star} \in \mathbb{S}^{3}$ one has $\mathcal{R}_{\tilde{Q}_{\star}} \in \mathbb{S O}(3)$. As such, in the algorithm setup it is not hard to obtain $\tilde{q}_{\star 0} \in \mathbb{R}_{+}$for all $t \geq 0$. Define the following positive time-decreasing prescribed performance function (PPF) with the map $\xi_{\star}: \mathbb{R}_{+} \rightarrow \mathbb{R}_{+}[20]$

$$
\xi_{\star}(t)=\left(\xi_{\star}^{0}-\xi_{\star}^{\infty}\right) \exp \left(-\ell_{\star} t\right)+\xi_{\star}^{\infty}
$$

with $\xi_{\star}(0)=\xi_{\star}^{0}>0$ and $\xi_{\star}^{\infty}>0$ being the upper bounds of a known large set and small set, respectively, and $\ell_{\star}>0$ being the convergence rate of $\xi_{\star}=\xi_{\star}(t)$ from $\xi_{\star}^{0}$ to $\xi_{\star}^{\infty}$. It can be deduced that $\lim _{t \rightarrow \infty} \xi_{\star}=\xi_{\star}^{\infty}$. $e_{\star}=e_{\star}(t)$ can be controlled by the predefined transient and steady-state boundaries provided that

$$
-\underline{\delta}_{\star} \xi_{\star}<e_{\star}<\xi_{\star}, \text { if } e_{\star}(0) \geq 0
$$

where $\underline{\delta}_{\star} \in[0,1]$. Due to the fact that $e_{\star} \in[0,1] \forall t \geq 0$, $e_{\star}$ is controlled by the PPF if the condition in (15) is met. Fig. 2 illustrates the concept of PPF in action allowing for the desired convergence of the constrained error $e_{\star}$ in (15).

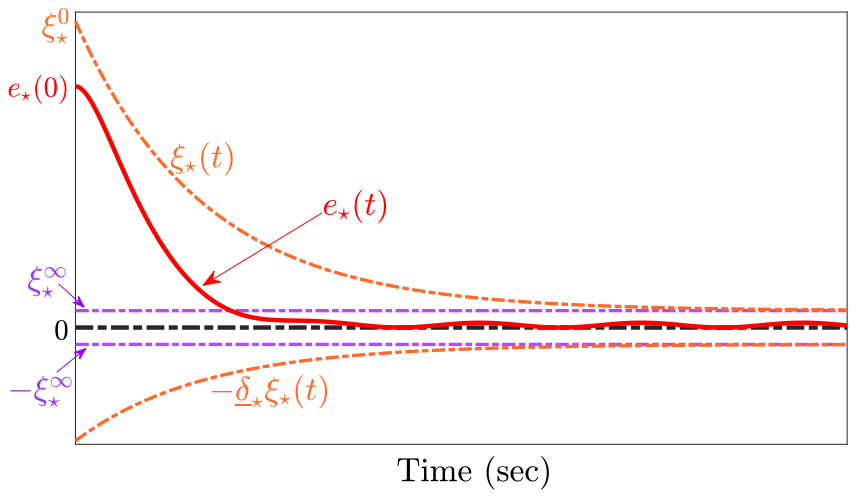

Fig. 2. Convergence of $e_{\star}(t)$ controlled by the PPF according to Eq. (15).

From (15) and Fig. 2, $e_{\star}$ is constrained. Let us redefine the constrained error $e_{\star}$ as

$$
e_{\star}=\xi_{\star} \mathcal{N}\left(E_{\star}\right)
$$

where $\xi_{\star}$ is given in (14), $E_{\star} \in \mathbb{R}$ is transformed (unconstrained) error, and $\mathcal{N}\left(E_{\star}\right)$ follows Assumption 2:

Assumption 2. $\mathcal{N}\left(E_{\star}\right)$ is characterized by the following properties [20]:

1. $\mathcal{N}\left(E_{\star}\right)$ is smooth and strictly increasing.

2. $\mathcal{N}\left(E_{\star}\right)$ is constrained by $-\underline{\delta}_{\star}<\mathcal{N}\left(E_{\star}\right)<\bar{\delta}_{\star}$ with $\bar{\delta}_{\star}$ and $\underline{\delta}_{\star}$ being positive constants and $\underline{\delta}_{\star} \leq \bar{\delta}_{\star}$.

3. $\lim _{E_{i} \rightarrow-\infty} \mathcal{N}\left(E_{\star}\right)=-\underline{\delta}_{i}$ and $\lim _{E_{i} \rightarrow+\infty} \mathcal{N}\left(E_{\star}\right)=\bar{\delta}_{i}$ where

$$
\mathcal{N}\left(E_{\star}\right)=\frac{\bar{\delta}_{\star} \exp \left(E_{\star}\right)-\underline{\delta}_{\star} \exp \left(-E_{\star}\right)}{\exp \left(E_{\star}\right)+\exp \left(-E_{\star}\right)}
$$

Based on (16) one finds

$$
E_{\star}=\mathcal{N}^{-1}\left(e_{\star} / \xi_{\star}\right)=\frac{1}{2} \ln \frac{\underline{\delta}_{\star}+e_{\star} / \xi_{\star}}{\bar{\delta}_{\star}-e_{\star} / \xi_{\star}}, \bar{\delta}_{\star} \geq \underline{\delta}_{\star}
$$

Remark 2. [2,27] It becomes apparent that selecting $\underline{\delta}_{\star}=\bar{\delta}_{\star}$ implies that $E_{\star}>0$ for all $e_{\star}>0$, and $E_{\star}=0$ only at $e_{\star}=0$. Thus, the critical point of $e_{\star}$ coincides with the critical point of $E_{\star}$.

Remark 3. [2,27] From (14), (16), and (18), $e_{\star}$ is constrained by $\xi_{\star}$ if and only if $E_{\star} \in \mathcal{L}_{\infty}$.

Define

$$
\begin{aligned}
\Delta_{\star} & =\frac{1}{2 \xi_{\star}} \frac{\partial \mathcal{N}^{-1}\left(e_{\star} / \xi_{\star}\right)}{\partial\left(e_{\star} / \xi_{\star}\right)} \\
& =\frac{1 /\left(2 \xi_{\star}\right)}{\underline{\delta}_{\star}+e_{\star} / \xi_{\star}}+\frac{1 /\left(2 \xi_{\star}\right)}{\bar{\delta}_{\star}-e_{\star} / \xi_{\star}}
\end{aligned}
$$

where $\Delta_{\star}$ is a positive function. Hence, one obtains

$$
\dot{E}_{\star}=\Delta_{\star}\left(\dot{e}_{\star}-\frac{\dot{\xi}_{\star}}{\xi_{\star}} e_{\star}\right)
$$

\section{ObServer-BASEd CONTROLleR With Guaranteed CONVERGENCE}

\section{A. Full State Observer with Guaranteed Performance}

Let $\hat{Q} \in \mathbb{S}^{3}$ and $\hat{\Omega} \in \mathbb{R}^{3}$ denote the estimates of $Q$ and $\Omega$, respectively. Consider the following attitude and angular 
velocity observer design:

$$
\left\{\begin{array}{l}
\dot{\hat{Q}}=\frac{1}{2} \hat{Q} \odot \overline{\hat{\Omega}+W_{\Omega}}=\frac{1}{2} \Gamma\left(\hat{\Omega}+W_{\Omega}\right) \hat{Q} \\
\hat{J} \dot{\hat{\Omega}}=[\hat{J} \hat{\Omega}]_{\times} \hat{\Omega}+\hat{\tau}+\hat{J}[\hat{\Omega}]_{\times} W_{\Omega}+W_{\tau} \\
W_{\Omega}=-k_{o}\left(E_{o} \Delta_{o}+1\right) \mathcal{R}_{\tilde{Q}_{o}} \tilde{q}_{o} \\
W_{\tau}=-\gamma_{o}\left(E_{o} \Delta_{o}+1\right) \mathcal{R}_{\tilde{Q}_{o}} \tilde{q}_{o}
\end{array}\right.
$$

where $\tilde{Q}_{o}=\left[\tilde{q}_{o 0}, \tilde{q}_{o}^{\top}\right]^{\top}=\hat{Q}^{-1} \odot Q_{y}$ denotes the unitquaternion error in observation, $Q_{y}$ stands for a reconstructed unit-quaternion obtained, for instance, by QUEST algorithm $[13,26], \mathcal{R}_{\tilde{Q}_{o}}$ is the attitude observation error, $\left[0, \hat{\tau}^{\top}\right]^{\top}=$ $\tilde{Q}_{o} \odot \bar{\tau} \odot \tilde{Q}_{o}^{-1}$, or more simply, $\hat{\tau}=\mathcal{R}_{\tilde{Q}_{o}} \tau$ denotes the torque input described in the observer frame, $\hat{J}=\mathcal{R}_{\tilde{Q}_{o}} J \mathcal{R}_{\tilde{Q}_{o}}^{\top}$ represents the inertia matrix described in the observer-frame, $e_{o}=1-\tilde{q}_{o 0}$, and $E_{o}=\frac{1}{2} \ln \frac{\delta_{o}+e_{o} / \xi_{o}}{\bar{\delta}_{o}-e_{o} / \xi_{o}}$ denotes the transformed error. Additionally, $\xi_{o}$ is the PPF defined in (14) where $\xi_{o}^{0}>e_{o}(0), W_{\Omega}$ and $W_{\tau}$ are correction factors, and $k_{o}, \gamma_{o}$, and $\underline{\delta}_{o}=\bar{\delta}_{o}>e_{o}(0)$ are positive constants.

Theorem 1. Consider the dynamics in (5) and the observer in (21). Let Assumption 1 hold true given that the condition in Remark 1 is met. Let $k_{o}, \gamma_{o}, \underline{\delta}_{o}=\bar{\delta}_{o}>e_{o}(0), \xi_{o}^{0}>e_{o}(0)$, and $\xi_{o}^{\infty}$ be positive constants. Then for $\left.E_{o}(0) \in \mathcal{L}_{\infty}, 1\right) E_{o}, e_{o}$, and $\hat{\Omega}$ are globally bounded, and 2) starting from any initial conditions, all $E_{o}, e_{o}$, and $\tilde{\Omega}_{o}$ converge asymptotically to the origin with $\lim _{t \rightarrow \infty} \tilde{q}_{o} \rightarrow 0_{3 \times 1}$ and $\lim _{t \rightarrow \infty} \tilde{q}_{o 0} \rightarrow \pm 1$.

Proof: Recall the error in (9), $\tilde{Q}_{o}=\hat{Q}^{-1} \odot Q$. From (5) and (21), one obtains

$$
\begin{aligned}
\dot{\tilde{Q}}_{o} & =\dot{\hat{Q}}^{-1} \odot Q+\hat{Q}^{-1} \odot \dot{Q} \\
& =-\frac{1}{2} \overline{\hat{\Omega}+W_{\Omega}} \odot \tilde{Q}_{o}+\frac{1}{2} \tilde{Q}_{o} \odot \bar{\Omega} \\
& =\frac{1}{2} \tilde{Q}_{o} \odot\left(\bar{\Omega}-\tilde{Q}_{o}^{-1} \odot \frac{\hat{\Omega}+W_{\Omega}}{\hat{\Omega}_{0}} \odot \tilde{Q}_{o}\right) \\
& =\frac{1}{2} \tilde{Q}_{o} \odot\left[\begin{array}{c}
0 \\
\tilde{\Omega}_{o}-\mathcal{R}_{\tilde{Q}_{o}}^{\top} W_{\Omega}
\end{array}\right]
\end{aligned}
$$

In view of (4) and (5), the mapping of (22) to $\mathbb{S O}(3)$ is

$$
\dot{\mathcal{R}}_{\tilde{Q}_{o}}=\mathcal{R}_{\tilde{Q}_{o}}\left[\tilde{\Omega}_{o}-\mathcal{R}_{\tilde{Q}_{o}}^{\top} W_{\Omega}\right]_{\times}
$$

Hence, the dynamics in (22) become

$$
\left[\begin{array}{c}
\dot{\tilde{q}}_{o 0} \\
\dot{\tilde{q}}_{o}
\end{array}\right]=\frac{1}{2}\left[\begin{array}{c}
-\tilde{q}_{o}^{\top} \\
\tilde{q}_{o 0} \mathbf{I}_{3}+\left[\tilde{q}_{o}\right]_{\times}
\end{array}\right]\left(\tilde{\Omega}_{o}-\mathcal{R}_{\tilde{Q}_{o}}^{\top} W_{\Omega}\right)
$$

As such, $\dot{E}_{o}$ in (20) is as follows:

$$
\dot{E}_{o}=\Delta_{o}\left(-\frac{1}{2} \tilde{q}_{o}^{\top}\left(\tilde{\Omega}_{o}-\mathcal{R}_{\tilde{Q}_{o}}^{\top} W_{\Omega}\right)-\frac{\dot{\xi}_{o}}{\xi_{o}}\left(1-\tilde{q}_{o 0}\right)\right)
$$

where $\Delta_{o}=\frac{1 /\left(2 \xi_{o}\right)}{\underline{\delta}_{o}+e_{o} / \xi_{o}}+\frac{1 /\left(2 \xi_{o}\right)}{\bar{\delta}_{o}-e_{o} / \xi_{o}}$ as expressed in (19). Recall $\tilde{\Omega}_{o}=\Omega-\mathcal{R}_{\tilde{Q}_{o}}^{\top} \hat{\Omega}$ as in (10). From (5), (21), and (23), one finds

$$
\begin{aligned}
& J \dot{\tilde{\Omega}}_{o}=J \dot{\Omega}-J \dot{\mathcal{R}}_{\tilde{Q}_{o}}^{\top} \hat{\Omega}-J \mathcal{R}_{\tilde{Q}_{o}}^{\top} \dot{\hat{\Omega}} \\
& =[J \Omega]_{\times} \Omega+\left(J\left[\tilde{\Omega}_{o}\right]_{\times}-\left[J \mathcal{R}_{\tilde{Q}_{o}}^{\top} \hat{\Omega}\right]_{\times}\right) \mathcal{R}_{\tilde{Q}_{o}}^{\top} \hat{\Omega}-\tilde{\mathcal{R}}_{\tilde{Q}_{o}}^{\top} W_{\tau} \\
& =S(\Omega) \tilde{\Omega}_{o}-\left[J \tilde{\Omega}_{o}\right]_{\times} \tilde{\Omega}_{o}-\mathcal{R}_{\tilde{Q}_{o}}^{\top} W_{\tau}
\end{aligned}
$$

such that

$$
\begin{aligned}
& {[J \Omega]_{\times} \Omega+\left(J\left[\tilde{\Omega}_{o}\right]_{\times}-\left[J \mathcal{R}_{\tilde{Q}_{o}}^{\top} \hat{\Omega}\right]_{\times}\right) \mathcal{R}_{\tilde{Q}_{o}}^{\top} \hat{\Omega}} \\
& =\left([J \Omega]_{\times}-J[\Omega]_{\times}-[\Omega]_{\times} J\right) \tilde{\Omega}_{o}-\left[J \tilde{\Omega}_{o}\right]_{\times} \tilde{\Omega}_{o} \\
& =S(\Omega) \tilde{\Omega}_{o}-\left[J \tilde{\Omega}_{o}\right]_{\times} \tilde{\Omega}_{o}
\end{aligned}
$$

where $S(\Omega)=[J \Omega]_{\times}-J[\Omega]_{\times}-[\Omega]_{\times} J$ is a skew symmetric matrix. Consider the following Lyapunov function candidate

$$
V_{o}=E_{o}^{2}+\left(1-\tilde{q}_{o 0}\right)+\frac{1}{2 \gamma_{\Omega}} \tilde{\Omega}_{o}^{\top} J \tilde{\Omega}_{o}
$$

In view of (24), (25), and (26), and with direct substitution of $W_{\Omega}$ and $W_{\tau}$ by their definitions in (21), one finds the time derivative of $V_{o}$ in (28) as follows:

$$
\begin{aligned}
\dot{V}_{o}= & -\left(E_{o} \Delta_{o}+1\right) \tilde{q}_{o}^{\top}\left(\tilde{\Omega}_{o}-\mathcal{R}_{\tilde{Q}_{o}}^{\top} W_{\Omega}\right)-\frac{2 E_{o} \Delta_{o} \dot{\xi}_{o}}{\xi_{o}}\left(1-\tilde{q}_{o 0}\right) \\
& +\frac{1}{\gamma_{o}} \tilde{\Omega}_{o}^{\top}\left(S(\Omega) \tilde{\Omega}_{o}-\left[J \tilde{\Omega}_{o}\right]_{\times} \tilde{\Omega}_{o}-\mathcal{R}_{\tilde{Q}_{o}}^{\top} W_{\tau}\right) \\
\leq & -k_{o}\left(E_{o}^{2} \Delta_{o}^{2}+1\right)\left\|\tilde{q}_{o}\right\|^{2}
\end{aligned}
$$

where $1-\tilde{q}_{o 0} \leq 1-\tilde{q}_{o 0}^{2}=\left\|\tilde{q}_{o}\right\|^{2}$, and since $\ell_{o}>\dot{\xi}_{o} / \xi_{o}, k_{o}$ is selected such that $k_{o} \geq \ell_{o}$. Note that $\tilde{\Omega}_{o}^{\top} S(\Omega) \tilde{\Omega}_{o}=0$ and $\tilde{\Omega}_{o}^{\top}\left[J \tilde{\Omega}_{o}\right]_{\times} \tilde{\Omega}_{o}=0$. By the definition of $E_{o}$ in (18), $\underline{\delta}_{o}=\bar{\delta}_{o}$ implies that $E_{o}>0$ for all $1>\left|\tilde{q}_{o 0}\right|$ and $E_{o}=0$ only at $\tilde{q}_{o 0}= \pm 1$, see Remark (2). Hence, $V_{o}$ is a non-increasing function indicating that $E_{o}, e_{o}$, and $\tilde{\Omega}_{o}$ are bounded, $\lim _{t \rightarrow \infty} \tilde{q}_{o}=0_{3 \times 1}$, and $\lim _{t \rightarrow \infty} \mathcal{R}_{\tilde{Q}_{o}}=\mathbf{I}_{3}$. Thus, $W_{\Omega}, W_{\tau} \rightarrow 0_{3 \times 1}$, and, based on Barbalat Lemma, $\ddot{\tilde{Q}}_{o}$ and $\ddot{E}_{o}$ are bounded by $\dot{\tilde{Q}}_{o} \rightarrow 0_{4 \times 1}$. From (22), one has $\tilde{\Omega}_{o} \rightarrow 0_{3 \times 1}$, and, based on Barbalat Lemma, $\ddot{\tilde{\Omega}}_{O}$ is bounded and $\dot{\tilde{\Omega}}_{o} \rightarrow 0_{3 \times 1}$ completing the proof. Consider the cross term $-\frac{d}{d t} \frac{\tilde{q}_{o 0}}{2 \delta_{o}} \tilde{\Omega}_{o}^{\top} \tilde{q}_{o}$ with the following derivative:

$$
\begin{aligned}
-\frac{d}{d t} & \frac{\tilde{q}_{o 0}}{2 \delta_{o}} \tilde{\Omega}_{o}^{\top} \tilde{q}_{o}=-\frac{1}{2 \delta_{o}} \dot{\tilde{\Omega}}_{o}^{\top} \tilde{q}_{o}-\frac{1}{2 \delta_{o}} \tilde{\Omega}_{o}^{\top} \dot{\tilde{q}}_{o}-\frac{\dot{\tilde{q}}_{o 0}}{2 \delta_{o}} \tilde{\Omega}_{o}^{\top} \tilde{q}_{o} \\
\leq & -\frac{1}{4 \delta_{o}}\left\|\tilde{\Omega}_{o}\right\|^{2}+\frac{c_{o 1}+E_{o} \Delta_{o} k_{o}}{\delta_{o}}\left\|\tilde{\Omega}_{o}\right\|\left\|\tilde{q}_{o}\right\| \| \\
& +\frac{c_{o 2}}{\delta_{o}}\left\|\tilde{q}_{o}\right\|^{2}
\end{aligned}
$$

where $\bar{c}_{J}=\bar{\lambda}\left(J^{-1}\right), \eta_{\Omega}=\sup _{t>0} S(\Omega), c_{o 1}=\frac{\eta_{\Omega} \bar{c}_{J}}{2}+$ $\frac{\eta_{\Omega_{o}} \bar{c}_{J}}{2}+\frac{\eta_{\Omega_{o}}}{2}$, and $c_{o 2}=\frac{\gamma_{o} \bar{c}_{J}}{2}$. Let $c_{m}=\max \left\{c_{o 1}, k_{o}\right\}$, and consider the following Lyapunov function candidate:

$$
\mathcal{L}_{o}=E_{o}^{2}+\underbrace{\left(1-\tilde{q}_{o 0}\right)+\frac{1}{2 \gamma_{\Omega}} \tilde{\Omega}_{o}^{\top} J \tilde{\Omega}_{o}-\frac{\tilde{q}_{o 0}}{2 \delta_{o}} \tilde{\Omega}_{o}^{\top} \tilde{q}_{o}}_{\mathcal{L}_{q o}}
$$

with $e_{o}=\left[\left\|\tilde{q}_{o 0}\right\|,\left\|\tilde{q}_{o}\right\|,\left\|\tilde{\Omega}_{o}\right\|\right]^{\top}$

$$
\begin{gathered}
e_{o}^{\top} \underbrace{\left[\begin{array}{ccc}
2 & 0 & -1 \\
0 & 2 & -\frac{1}{4 \delta_{o}} \\
-1- & \frac{1}{4 \delta_{o}} & \frac{\underline{\lambda}_{J}}{2 \gamma_{\Omega}}
\end{array}\right]}_{P_{1}} e_{o} \leq \mathcal{L}_{q o} \\
\leq e_{o}^{\top} \underbrace{\left[\begin{array}{ccc}
2 & 0 & 1 \\
0 & 2 & \frac{1}{4 \delta_{o}} \\
1 & \frac{1}{4 \delta_{o}} & \frac{\lambda_{J}}{2 \gamma_{\Omega}}
\end{array}\right]}_{P_{2}} e_{o}
\end{gathered}
$$


$P_{1}$ and $P_{2}$ can be made positive by selecting $\underline{\lambda}_{J} \frac{16 \delta_{o}^{2}}{1+32 \delta_{o}^{2}}>$ $\gamma_{\Omega}$. From (29) and (30), and selecting $\delta_{o}>\frac{2 c_{o 2}}{c_{\ell}}$, the time derivative of $\mathcal{L}_{o}$ in $(31)$ is

$$
\dot{\mathcal{L}}_{o} \leq-\varepsilon_{o}^{\top} \underbrace{\left[\begin{array}{ccc}
k_{o} & 0 & \frac{c_{m}}{2 \delta_{o}} \\
0 & k_{o} & \frac{c_{m}}{2 \delta_{o}} \\
\frac{c_{m}}{2 \delta_{o}} & \frac{c_{m}}{2 \delta_{o}} & \frac{1}{4 \delta_{o}}
\end{array}\right]}_{A_{o}} \varepsilon_{o}
$$

where $\varepsilon_{o}=\left[\left\|\tilde{q}_{o}\right\|, E_{o} \Delta_{o}\left\|\tilde{q}_{o}\right\|,\left\|\tilde{\Omega}_{o}\right\|\right]^{\top}$. $A_{o}$ can be made positive if $\delta_{o}>\frac{2 c_{m}^{2}}{k_{o}}$ such that

$$
\dot{\mathcal{L}}_{o} \leq-\underline{\lambda}_{o}\left(E_{o}^{2} \Delta_{o}^{2}+1\right)\left\|\tilde{q}_{o}\right\|^{2}-\underline{\lambda}_{o}\left\|\tilde{\Omega}_{o}\right\|^{2}
$$

with $\underline{\lambda}_{o}=\underline{\lambda}\left(A_{o}\right)$ being the minimum singular value of $A_{o}$ proving Theorem 1.

\section{B. Observer-based Controller with Guaranteed Convergence}

Consider the following controller design with control input $\tau$ for (5):

$$
\left\{\begin{aligned}
\dot{Q}_{a}= & \frac{1}{2} Q_{a} \odot \overline{\beta_{a}}=\frac{1}{2} \Gamma\left(\beta_{a}\right) Q_{a}, \quad Q_{a}(0) \in \mathbb{S}^{3} \\
\beta_{a}= & -k_{\beta}\left(E_{a} \Delta_{a}+1\right) \mathcal{R}_{\tilde{Q}_{a}}^{\top} \tilde{q}_{a} \\
W_{c}= & -k_{w}\left(E_{a} \Delta_{a} \tilde{q}_{a}+\tilde{q}_{c}\right) \\
\tau= & -W_{c}-k_{c}\left(\mathcal{R}_{\tilde{Q}_{o}}^{\top} \hat{\Omega}_{-}-\mathcal{R}_{\tilde{Q}_{c}}^{\top} \Omega_{d}\right) \\
& +\left[\mathcal{R}_{\tilde{Q}_{c}}^{\top} \Omega_{d}\right]_{\times} J \mathcal{R}_{\tilde{Q}_{c}}^{\top} \Omega_{d}+J \mathcal{R}_{\tilde{Q}_{c}}^{\top} \dot{\Omega}_{d}
\end{aligned}\right.
$$

where $\tilde{Q}_{c}=\left[\tilde{q}_{c 0}, \tilde{q}_{c}^{\top}\right]^{\top}=Q_{d}^{-1} \odot Q$ is the unit-quaternion error, $Q_{d}$ is the desired unit-quaternion, $Q_{a}=\left[q_{a 0}, q_{a}^{\top}\right]^{\top}$ is the auxiliary unit-quaternion, $\tilde{Q}_{a}=\left[\tilde{q}_{a 0}, \tilde{q}_{a}^{\top}\right]^{\top}=Q_{a}^{-1} \odot \tilde{Q}_{c}$, $\mathcal{R}_{\tilde{Q}_{c}}$ is the attitude control error, $\mathcal{R}_{\tilde{Q}_{a}}$ is the attitude auxiliary error, $e_{a}=1-\tilde{q}_{a 0}, E_{a}=\frac{1}{2} \ln \frac{\underline{\delta}_{a}+e_{a} / \xi_{a}}{\bar{\delta}_{a}-e_{a} / \xi_{a}}$ is the transformed error, $W_{c}$ and $\beta_{a}$ are the correction factors, and $\dot{\Omega}_{d}$ is the derivative of the desired angular velocity. Additionally, $\xi_{a}$ is the PPF defined in (14) with $\xi_{a}^{0}>e_{a}(0)$, and $k_{w}, k_{c}, k_{\beta}$, and $\underline{\delta}_{a}=\bar{\delta}_{a}>e_{a}(0)$ are positive constants.

Theorem 2. Consider the dynamics in (5) and the control law in (34). Let Assumption 1 hold. Let the design parameters $k_{w}$, $k_{c}, k_{\beta}, \bar{\delta}_{a}=\underline{\delta}_{a}>e_{a}(0), \xi_{a}^{0}>e_{a}(0)$, and $\xi_{a}^{\infty}$ be positive constants with $E_{a}(0) \in \mathcal{L}_{\infty}$. Then, 1) $E_{a}, e_{a}$, and $\Omega$ are globally bounded, and 2) starting from any initial conditions, all $E_{a}, e_{a}$, and $\tilde{\Omega}_{c}$ converge asymptotically to the origin with $\lim _{t \rightarrow \infty} \tilde{q}_{a} \rightarrow 0_{3 \times 1}, \lim _{t \rightarrow \infty} \tilde{q}_{a 0} \rightarrow \pm 1 \lim _{t \rightarrow \infty} \tilde{q}_{c} \rightarrow 0_{3 \times 1}$, and $\lim _{t \rightarrow \infty} \tilde{q}_{c 0} \rightarrow \pm 1$.

Proof: Consider $\tilde{Q}_{c}=Q_{d}^{-1} \odot Q$ as in (11). From (5) and (8), one finds

$$
\begin{aligned}
\dot{\tilde{Q}}_{c} & =\frac{1}{2} \tilde{Q}_{c} \odot\left(\bar{\Omega}-\tilde{Q}_{c}^{-1} \odot \overline{\Omega_{d}} \odot \tilde{Q}_{c}\right) \\
& =\frac{1}{2} \tilde{Q}_{c} \odot\left[0, \tilde{\Omega}_{c}^{\top}\right]^{\top} \\
\dot{\mathcal{R}}_{\tilde{Q}_{c}} & =\mathcal{R}_{\tilde{Q}_{c}}\left[\tilde{\Omega}_{c}\right]_{\times}
\end{aligned}
$$

Hence, in view of (22) and (24), one finds that $\dot{\tilde{q}}_{c 0}=$ $-\frac{1}{2} \tilde{q}_{c}^{\top} \tilde{\Omega}_{c}$ and $\dot{\tilde{q}}_{c}=\frac{1}{2}\left(\tilde{q}_{c 0} \mathbf{I}_{3}+\left[\tilde{q}_{c}\right]_{\times}\right) \tilde{\Omega}_{c}$. In the same spirit, $\dot{\tilde{Q}}_{a}=\frac{1}{2} \tilde{Q}_{c} \odot\left[0,\left(\tilde{\Omega}_{c}-\mathcal{R}_{\tilde{Q}_{a}}^{\top} \beta_{a}\right)^{\top}\right]^{\top}$ such that $\dot{\mathcal{R}}_{\tilde{Q}_{a}}=$
$\mathcal{R}_{\tilde{Q}_{a}}\left[\tilde{\Omega}_{c}-\mathcal{R}_{\tilde{Q}_{a}}^{\top} \beta_{a}\right]_{\times}$. Thus, the transformed error dynamics of $E_{a}$ become

$$
\dot{E}_{a}=\Delta_{a}\left(-\frac{1}{2} \tilde{q}_{a}^{\top}\left(\tilde{\Omega}_{c}-\mathcal{R}_{\tilde{Q}_{a}}^{\top} \beta_{a}\right)-\frac{\dot{\xi}_{a}}{\xi_{a}}\left(1-\tilde{q}_{a 0}\right)\right)
$$

where $\Delta_{a}=\frac{1 /\left(2 \xi_{a}\right)}{\underline{\delta}_{a}+e_{a} / \xi_{a}}+\frac{1 /\left(2 \xi_{a}\right)}{\delta_{a}-e_{a} / \xi_{a}}$ as specified in (19). Let $\tilde{\Omega}_{c}=\Omega-\mathcal{R}_{\tilde{Q}_{c}}^{\top} \Omega_{d}$ as defined in (12). In view of the steps in (26) and from (5), (34), and (36), one has

$$
J \dot{\tilde{\Omega}}_{c}=S(\Omega) \tilde{\Omega}_{c}-\left[J \tilde{\Omega}_{c}\right]_{\times} \tilde{\Omega}_{c}-W_{c}-k_{c}\left(\tilde{\Omega}_{c}-\tilde{\Omega}_{o}\right)
$$

with $[J \Omega]_{\times} \Omega+J\left[\tilde{\Omega}_{c}\right]_{\times} \mathcal{R}_{\tilde{Q}_{c}}^{\top} \Omega_{d}+\left[\mathcal{R}_{\tilde{Q}_{c}}^{\top} \Omega_{d}\right]_{\times} J \mathcal{R}_{\tilde{Q}_{c}}^{\top} \Omega_{d}=$ $S(\Omega) \tilde{\Omega}_{c}-\left[J \tilde{\Omega}_{c}\right]_{\times} \tilde{\Omega}_{c}, S(\Omega)=[J \Omega]_{\times}-J[\Omega]_{\times}-[\Omega]_{\times} J$ being a skew symmetric matrix, see (27). Consider the following Lyapunov function candidate

$$
\mathcal{L}_{c}=E_{a}^{2}+2\left(1-\tilde{q}_{c 0}\right)+\frac{1}{2 k_{w}} \tilde{\Omega}_{c}^{\top} J \tilde{\Omega}_{c}
$$

From (35), (37), and (38), and replacing $W_{c}$ and $\beta_{a}$ with their definitions in (34) one finds the time derivative of $\mathcal{L}_{c}$ in (39) as follows:

$$
\begin{aligned}
\dot{\mathcal{L}}_{c} & =-E_{a} \Delta_{a} \tilde{q}_{a}^{\top}\left(\tilde{\Omega}_{c}-\mathcal{R}_{\tilde{Q}_{a}}^{\top} \beta_{a}\right)-\tilde{q}_{c}^{\top} \tilde{\Omega}_{c} \\
& -\frac{2 E_{a} \Delta_{a} \dot{\xi}_{a}}{\xi_{a}}\left(1-\tilde{q}_{a 0}\right) \\
& +\frac{1}{k_{w}} \tilde{\Omega}_{c}^{\top}\left(S(\Omega) \tilde{\Omega}_{c}-\left[J \tilde{\Omega}_{c}\right]_{\times} \tilde{\Omega}_{c}-W_{c}-k_{c}\left(\tilde{\Omega}_{c}-\tilde{\Omega}_{o}\right)\right) \\
\leq & -k_{\beta} E_{a}^{2} \Delta_{a}^{2}\left\|\tilde{q}_{a}\right\|^{2}-\frac{k_{c}}{k_{w}}\left\|\tilde{\Omega}_{c}\right\|^{2}+\frac{k_{c}}{k_{w}}\left\|\tilde{\Omega}_{c}\right\|\left\|\tilde{\Omega}_{o}\right\|
\end{aligned}
$$

where $2 E_{a} \Delta_{a} \frac{\dot{\xi}_{a}}{\xi_{a}}\left(1-\tilde{q}_{a 0}\right) \leq 2 \ell_{a} E_{a} \Delta_{a}\left\|\tilde{q}_{a}\right\|^{2}$ and $k_{\beta}$ is selected such that $k_{\beta} \geq 2 \ell_{a}$. According to the skew symmetric definition, $\tilde{\Omega}_{c}^{\top} S(\Omega) \tilde{\Omega}_{c}=0$ and $\tilde{\Omega}_{c}^{\top}\left[J \tilde{\Omega}_{c}\right]_{\times} \tilde{\Omega}_{c}=0$. Let us combine (31) and (39) to obtain the following Lyapunov function candidate:

$$
\mathcal{L}_{T}=\mathcal{L}_{o}+\mathcal{L}_{c}
$$

From (33) and (40), one finds

$$
\begin{aligned}
\dot{\mathcal{L}}_{T} \leq & -\underline{\lambda}_{o}\left(E_{o}^{2} \Delta_{o}^{2}+1\right)\left\|\tilde{q}_{o}\right\|^{2}-k_{\beta} E_{a}^{2} \Delta_{a}^{2}\left\|\tilde{q}_{a}\right\|^{2} \\
& -\varepsilon_{\Omega}^{\top} \underbrace{\left[\begin{array}{cc}
\lambda_{o} & 0.5 k_{c} / k_{w} \\
0.5 k_{c} / k_{w} & k_{c} / k_{w}
\end{array}\right]}_{A_{\Omega}} \varepsilon_{\Omega}
\end{aligned}
$$

where $\varepsilon_{\Omega}=\left[\left\|\tilde{\Omega}_{o}\right\|,\left\|\tilde{\Omega}_{c}\right\|\right]^{\top}$. $A_{\Omega}$ can be made positive by selecting $\underline{\lambda}_{o}>\frac{k_{c}}{4 k_{w}}$. Let $\underline{\lambda}_{c}=\underline{\lambda}\left(A_{\Omega}\right)$ be the minimum eigenvalue of $A_{\Omega}$. One obtains

$$
\begin{aligned}
\dot{\mathcal{L}}_{T} \leq & -\underline{\lambda}_{o}\left(E_{o}^{2} \Delta_{o}^{2}+1\right)\left\|\tilde{q}_{o}\right\|^{2}-k_{\beta} E_{a}^{2} \Delta_{a}^{2}\left\|\tilde{q}_{a}\right\|^{2}-\underline{\lambda}_{c}\left\|\tilde{\Omega}_{o}\right\| \\
& -\underline{\lambda}_{c}\left\|\tilde{\Omega}_{c}\right\|
\end{aligned}
$$

The inequality in (43) shows that $\ddot{\mathcal{L}}_{T}$ is bounded and $\dot{\mathcal{L}}_{T}$ goes to zero proving Theorem 2 in addition to $\tilde{q}_{a} \rightarrow 0_{3 \times 1}$ and $\tilde{\Omega}_{c} \rightarrow 0_{3 \times 1}$. Since $\underline{\delta}_{a}=\bar{\delta}_{a}, E_{a} \neq 0$ for all $e_{a} \neq 0$ and $E_{a}=0$ only at $e_{a}=0$. Thus, $e_{a}, E_{a} \rightarrow 0$ which implies that $\tilde{q}_{a} \rightarrow 0_{3 \times 1}$ and $\tilde{q}_{a 0} \rightarrow \pm 1$. Based on Barbalat Lemma, $\ddot{\tilde{\Omega}}_{c}$ and $\ddot{\tilde{Q}}_{a}$ are bounded with $\dot{\tilde{\Omega}}_{c} \rightarrow 0_{3 \times 1}$ and $\dot{\tilde{Q}}_{a} \rightarrow 0_{3 \times 1}$. From (35), $\tilde{\Omega}_{c} \rightarrow 0_{3 \times 1}$ indicates that $\dot{\tilde{Q}}_{c} \rightarrow 0_{4 \times 1}$. Moreover, from 


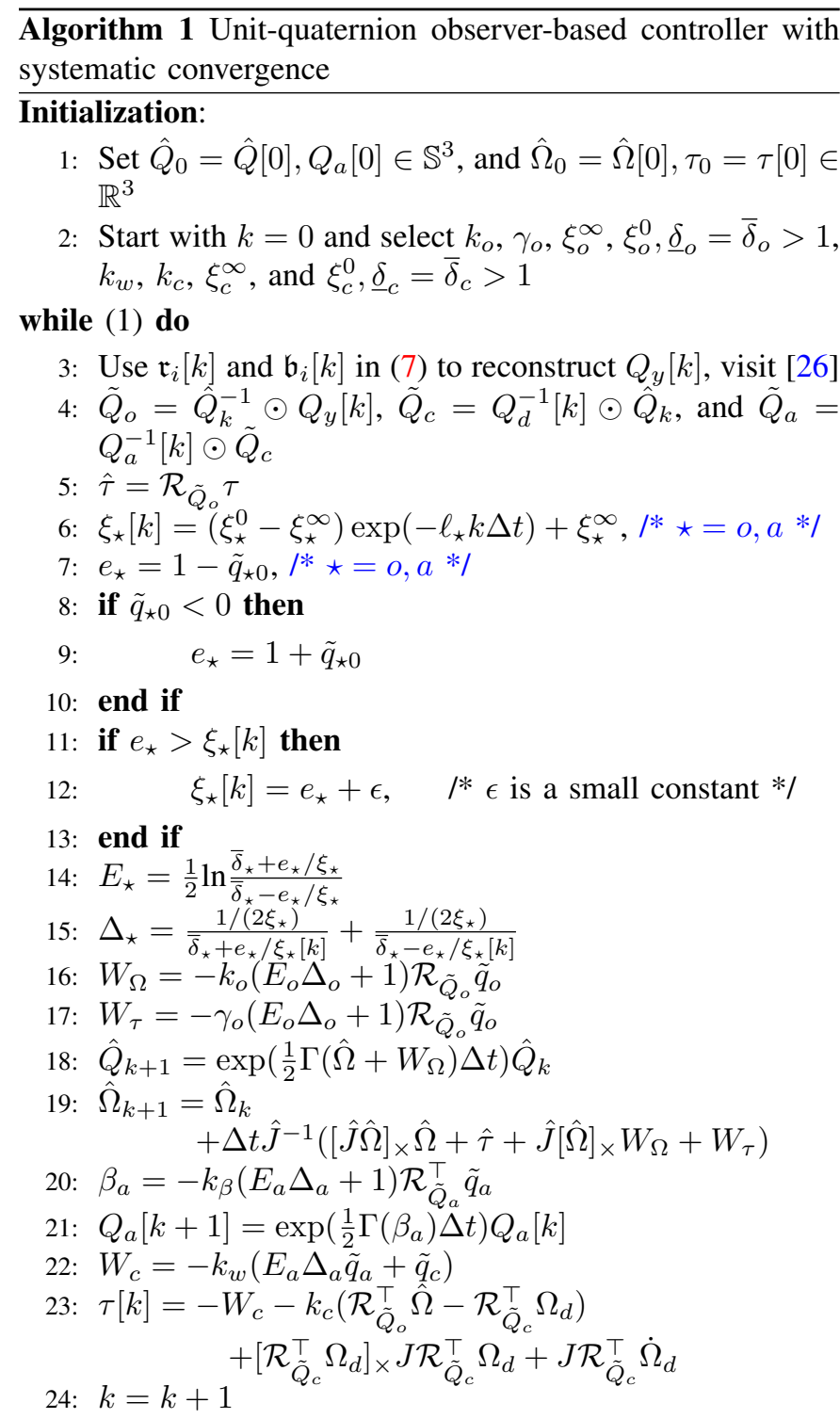

end while

(38), $J \dot{\tilde{\Omega}}_{c} \rightarrow 0_{3 \times 1}$ shows that $W_{c} \rightarrow 0_{3 \times 1}$ which shows that $\tilde{q}_{c} \rightarrow 0_{3 \times 1}$ and, in turn, $\tilde{q}_{c 0} \rightarrow \pm 1$ completing the proof.

Let $\Delta t$ be a small sample time. Algorithm 1 lists the complete implementation steps of a discrete form of the proposed quaternion observer-based controller with guaranteed performance.

\section{NumERiCAL RESUlts}

This section reveals the robustness of of the novel guaranteed performance discrete quaternion observer-based controller described in Algorithm 1 at low sampling rate of $200 \mathrm{~Hz}$. Let $r_{1}=[1,1.2,1.3]^{\top}$ and $r_{2}=[0,0,1]^{\top}$ be two noncollinear inertial-frame vectors with body-frame measured values being corrupted by zero-mean noise with a standard deviation of 0.08 , visit (6). Based on Remark 1, $\mathfrak{r}_{i}=r_{i} /\left\|r_{i}\right\|$ and $\mathfrak{b}_{i}=b_{i} /\left\|b_{i}\right\|$ for $i=1,2$ with $\mathfrak{r}_{3}=\mathfrak{r}_{1} \times \mathfrak{r}_{2}$ and $\mathfrak{b}_{3}=$ $\mathfrak{b}_{1} \times \mathfrak{b}_{2}$. Let $\Omega(0)=[0.2,0.3,0.3]^{\top}$ and its initial estimate be $\hat{\Omega}(0)=[0,0,0]^{\top}$. In order to account for a large initial error of the unit-quaternion between $Q[0]$ and $\hat{Q}[0]$ and between $Q[0]$ and $Q_{d}[0]$, consider $Q[0]=[0.0087,0.3906,0.1302,0.9113]^{\top}$, $Q_{d}[0]=Q_{a}[0]=\hat{Q}[0]=[1,0,0,0]^{\top}$. Assume that the rigidbody's inertia matrix is $J=\operatorname{diag}(0.016,0.015,0.03)$. Let the time derivative of the desired angular velocity be

$$
\dot{\Omega}_{d}=\left[\begin{array}{c}
0.03 \sin (0.3 t+\pi / 4) \\
0.05 \sin (0.4 t+\pi / 3) \\
0.02 \sin (0.2 t+\pi / 2)
\end{array}\right] \mathrm{rad} / \mathrm{sec}^{2}
$$

Select the design parameters as follows: $\xi_{o}^{0}=\xi_{a}^{0}=\bar{\delta}_{o}=\underline{\delta}_{o}=$ $\bar{\delta}_{a}=\underline{\delta}_{a}=1.7, \xi_{o}^{\infty}=\xi_{a}^{\infty}=0.05, \ell_{o}=\ell_{a}=1, k_{o}=10$, $k_{w}=1$, and $\gamma_{o}=k_{c}=k_{\beta}=0.1$.

For simplicity in demonstration, Fig. 3 shows the third component of the body-frame measurements with respect to the true values. Despite high noise level in the body-frame measurements of an IMU module illustrated by Fig. 3, Fig. 4 reveals impressive tracking capabilities in case of large initialization error and fast maneuvering. The robustness and fast adaptation of the proposed approach are confirmed in Fig. 5 where attitude tracking errors are successfully regulated to the desired equilibrium point $\mathbf{Q}_{\mathrm{I}}=[ \pm 1,0,0,0]$ and angular velocity tracking errors are regulated to the origin. Furthermore, Fig. 6 demonstrates the boundedness of the control signal. Note that unit-quaternion is subject to non-uniqueness such that for $Q_{1}=-Q_{2} \in \mathbb{S}^{3}, \mathcal{R}_{Q_{1}}=\mathcal{R}_{Q_{2}} \in \mathbb{S O}(3)$. Only for plotting purposes, if $\tilde{q}_{c 0} \rightarrow \pm 1$ and $\tilde{q}_{o 0} \rightarrow \mp 1$, multiply $\hat{Q}$ by -1 to end with $\tilde{q}_{o 0} \rightarrow \pm 1$.

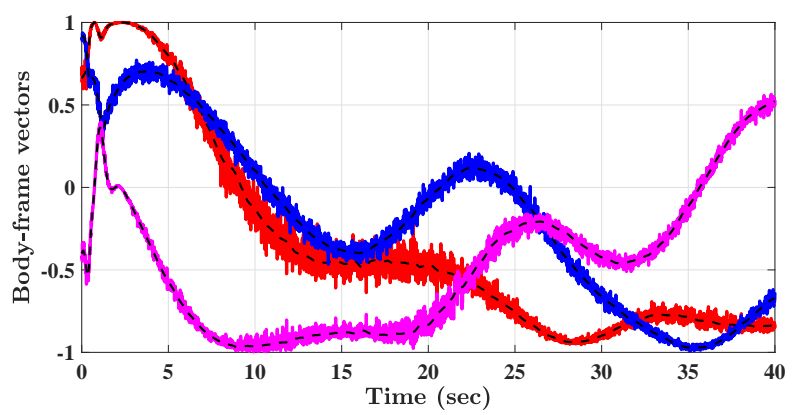

Fig. 3. Body-frame vectors: Measurements (red, blue, and magenta solidlines) vs true (black dashed-line).
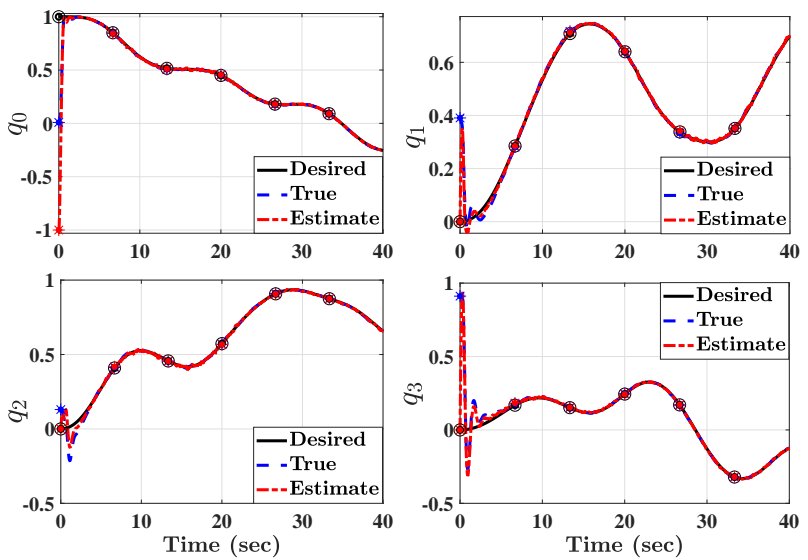

Fig. 4. Unit-quaternion: desired $Q_{d}$, true $Q$, and estimated $\hat{Q}$. 

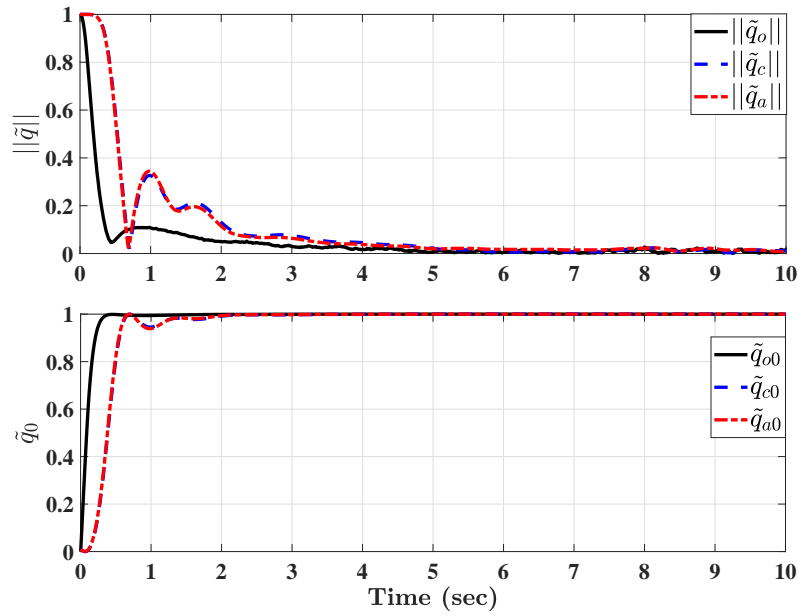

Fig. 5. Errors in unit-quaternion: $\tilde{Q}_{o}, \tilde{Q}_{c}$, and $\tilde{Q}_{a}$.
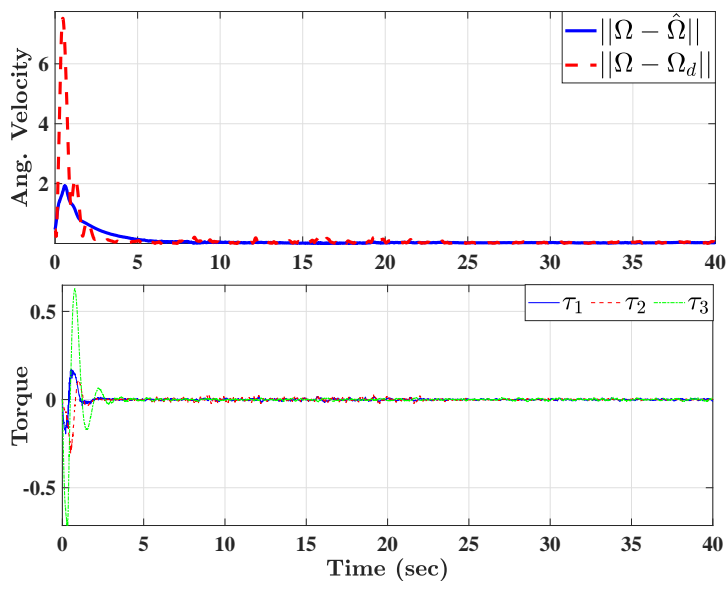

Fig. 6. Control input (torque) and errors in angular velocity.

\section{CONCLUSION}

This paper addressed the challenge of velocity-free attitude tracking performed based solely on measurements obtained from low-cost inertial measurement units. A novel computationally cheap unit-quaternion observer-based controller ensuring almost global asymptotic stability of the overall closed loop signals has been proposed. Additionally, the transient and steady-state performance of the attitude tracking error has been shown to follow the dynamically reducing boundaries predefined by the user. Simulation results demonstrated high robustness and fast adaptation at a low sampling rate.

\section{ACKNOWLEDGMENT}

The author would like to thank Maria Shaposhnikova for proofreading the article.

\section{REFERENCES}

[1] S. Salcudean, "A globally convergent angular velocity observer for rigid body motion," IEEE transactions on Automatic Control, vol. 36, no. 12, pp. 1493-1497, 1991.

[2] H. A. Hashim, "Systematic convergence of nonlinear stochastic estimators on the special orthogonal group $\mathrm{SO}(3)$," International Journal of Robust and Nonlinear Control, vol. 30, no. 10, pp. 3848-3870, 2020.
[3] F. Lizarralde and J. T. Wen, "Attitude control without angular velocity measurement: A passivity approach," IEEE transactions on Automatic Control, vol. 41, no. 3, pp. 468-472, 1996.

[4] C. G. Mayhew, R. G. Sanfelice, and A. R. Teel, "Quaternion-based hybrid control for robust global attitude tracking," IEEE Transactions on Automatic control, vol. 56, no. 11, pp. 2555-2566, 2011.

[5] H. A. Hashim, M. Abouheaf, and M. A. Abido, "Geometric stochastic filter with guaranteed performance for autonomous navigation based on IMU and feature sensor fusion," Control Engineering Practice, vol. PP, no. PP, pp. 1-11, 2021.

[6] H. A. Hashim, L. J. Brown, and K. McIsaac, "Nonlinear stochastic attitude filters on the special orthogonal group 3: Ito and stratonovich," IEEE Transactions on Systems, Man, and Cybernetics: Systems, vol. 49, no. 9, pp. 1853-1865, 2019.

[7] A. Castillo, R. Sanz, P. Garcia, W. Qiu, H. Wang, and C. Xu, "Disturbance observer-based quadrotor attitude tracking control for aggressive maneuvers," Control Engineering Practice, vol. 82, pp. 14-23, 2019.

[8] H. A. Hashim, "A geometric nonlinear stochastic filter for simultaneous localization and mapping," Aerospace Science and Technology, vol. 111, p. 106569, 2021.

[9] B. Cui, Y. Xia, K. Liu, Y. Wang, and D.-H. Zhai, "Velocity-observerbased distributed finite-time attitude tracking control for multiple uncertain rigid spacecraft," IEEE Transactions on Industrial Informatics, vol. 16, no. 4, pp. 2509-2519, 2019.

[10] H. A. Hashim, "GPS-denied navigation: Attitude, position, linear velocity, and gravity estimation with nonlinear stochastic observer," in 2021 American Control Conference (ACC). IEEE, 2021, pp. 1146-1151.

[11] H. A. Hashim and A. E. E. Eltoukhy, "Nonlinear filter for simultaneous localization and mapping on a matrix Lie Group using IMU and feature measurements," IEEE Transactions on Systems, Man, and Cybernetics: Systems, vol. PP, no. PP, pp. 1-12, 2021.

[12] "Nasa's hubble space telescope returns to science operations," NASA TV, 2018. [Online]. Available: https://www.nasa.gov/hubble

[13] M. D. Shuster and S. D. Oh, "Three-axis attitude determination from vector observations," Journal of Guidance, Control, and Dynamics, vol. 4, pp. 70-77, 1981.

[14] A. Odry, R. Fuller, I. J. Rudas, and P. Odry, "Kalman filter for mobilerobot attitude estimation: Novel optimized and adaptive solutions," Mechanical systems and signal processing, vol. 110, pp. 569-589, 2018.

[15] A. Odry, "An open-source test environment for effective development of marg-based algorithms," Sensors, vol. 21, no. 4, p. 1183, 2021.

[16] F. Caccavale and L. Villani, "Output feedback control for attitude tracking," Systems \& Control Letters, vol. 38, no. 2, pp. 91-98, 1999.

[17] B. Costic, D. Dawson, M. De Queiroz, and V. Kapila, "Quaternion-based adaptive attitude tracking controller without velocity measurements," Journal of Guidance, Control, and Dynamics, vol. 24, no. 6, pp. 12141222, 2001.

[18] Y. Xia, Z. Zhu, M. Fu, and S. Wang, "Attitude tracking of rigid spacecraft with bounded disturbances," IEEE Transactions on Industrial Electronics, vol. 58, no. 2, pp. 647-659, 2010

[19] M. Tognon and A. Franchi, "Dynamics, control, and estimation for aerial robots tethered by cables or bars," IEEE Transactions on Robotics, vol. 33, no. 4, pp. 834-845, 2017.

[20] C. P. Bechlioulis and G. A. Rovithakis, "Robust adaptive control of feedback linearizable mimo nonlinear systems with prescribed performance," IEEE Transactions on Automatic Control, vol. 53, no. 9, pp. 2090-2099, 2008

[21] C. Zhang, G. Ma, Y. Sun, and C. Li, "Observer-based prescribed performance attitude control for flexible spacecraft with actuator saturation," ISA transactions, vol. 89, pp. 84-95, 2019.

[22] Z. Yin, A. Suleman, J. Luo, and C. Wei, "Appointed-time prescribed performance attitude tracking control via double performance functions," Aerospace Science and Technology, vol. 93, p. 105337, 2019.

[23] H. A. Hashim, "Special orthogonal group $\mathrm{SO}(3)$, euler angles, angleaxis, rodriguez vector and unit-quaternion: Overview, mapping and challenges," arXiv preprint arXiv:1909.06669, 2019.

[24] M. D. Shuster, "A survey of attitude representations," Navigation, vol. 8, no. 9, pp. 439-517, 1993.

[25] D. Mortari, "Second estimator of the optimal quaternion," Journal of Guidance, Control, and Dynamics, vol. 23, no. 5, pp. 885-888, 2000.

[26] H. A. Hashim, "Attitude determination and estimation using vector observations: Review, challenges and comparative results," arXiv preprint arXiv:2001.03787, 2020.

[27] H. A. Hashim and A. E. E. Eltoukhy, "Landmark and IMU data fusion: Systematic convergence geometric nonlinear observer for SLAM and velocity bias," IEEE Transactions on Intelligent Transportation Systems, vol. PP, no. PP, pp. 1-10, 2020. 